\title{
AMBIENTALIZAR LA UNIVERSIDAD: UN RETO INSTITUCIONAL PARA EL ASEGURAMIENTO DE LA CALIDAD EN LOS ÁMBITOS CURRICULARES Y DE LA GESTIÓN
}

\author{
José Gutiérrez Pérez \\ Universidad de Granada, España. \\ Alexis González Dulzaides \\ Universidad de Ciego de Ávila, Cuba
}

\section{INTRODUCCIÓN}

La humanidad ha entrado en el siglo XXI en medio de una profunda crisis ambiental, reflejada no sólo en el ámbito natural, como la desertificación, el deterioro de la capa de ozono, las sequías, etc, sino también en el plano social al acrecentarse el desempleo, las migraciones forzadas y masivas, la pobreza y las diferencias entre regiones y grupos sociales. El modelo de sociedad industrial actualmente imperante ha conseguido instaurarse a escala planetaria, acentuando un doble efecto de situaciones indeseables: el de crisis social, como consecuencia de la pobreza material y moral de los seres humanos; y el de crisis ecológica, como consecuencia del deterioro de ecosistemas, transformación de condiciones de los biotopos y destrucción o extinción de biocenosis.

El ser humano ha adquirido paulatinamente conciencia de que tiene ante sí una disyuntiva que plantea dos opciones inequívocas: por un lado continuar con modelos de desarrollo en que los procesos económicos prevalecen y marcan las líneas de explotación de los recursos y hábitos de consumo, o en necesario contraste, entender que si alguna dictadura debe existir en este siglo, esa es la dictadura ambiental (Guillén, 1998). El paradigma del desarrollo sostenible constituye hoy una alternativa consistente y esperanzadora a las crisis actuales, en que se promulga una reorientación de los modelos económicos y tecnológicos inspirados en reorientaciones culturales y axiológicas que se sobrepongan a planteamientos exclusivamente antropocéntricos. La reorientación de modelos exige también una recomposición de las formulaciones epistemológicas y de las lógicas disciplinares en que se articulan los actuales conocimientos científicos para abrir paso a otros escenarios de integración interdisciplinar y construcción participada de la ciencia básica y aplicada.

Sin embargo, desde los años setenta el hombre comenzó a tomar conciencia de la relación existente entre los fenómenos antes mencionados y el sistema socioeconómico imperante, es por ello, que diversas ramas de la ciencia, entre ellas la educación, han tratado de diseñar estrategias alternativas en la búsqueda de un nuevo modelo de desarrollo caracterizado por la igualdad, la durabilidad, la eficiencia, la redistribución, la equidad, la suficiencia y la solidaridad. Gutiérrez (2004) plantea al respecto que: "Conseguir una sociedad cada vez más comprometida con el medio ambiente es un reto ambicioso que exige reformas e innovaciones en lo privado y en lo público, en lo personal y en lo institucional, en lo individual y lo colectivo, en lo educativo y en las demás esferas de la vida ciudadana. En este sentido, las instituciones educativos de las sociedades modernas, no sólo tienen el deber de incorporar estrategias para conseguir ciudadanos ambientalmente educados; además tienen la responsabilidad de predicar con el ejemplo, desarrollando actuaciones modélicas que incorporen a sus estructuras organizativas nuevos modelos de gestión y nuevas formas de aprovechamiento alternativo de los recursos". 
No se trata por tanto de una educación para o a favor del medio ambiente, sino una educación para cambiar la sociedad, una educación total que contribuya a la mejora de la calidad de vida de las personas y de su entorno y que se centre en el sujeto de la educación y no en el medio ambiente. Las escuelas, los centros de educación secundaria, las universidades y las instituciones educativas en general, pueden ser un interesante vehículo para favorecer el desarrollo sostenible de los pueblos, de las ciudades y de las sociedades modernas. A menos que no empecemos por cambiar cosas tan elementales como los hábitos cotidianos, las acciones rutinarias de nuestro quehacer diario, o las pequeñas decisiones que a menudo tomamos en nuestros hogares, en nuestros hábitos de consumo, en nuestro trabajo, en nuestro tiempo de ocio, en nuestros centros educativos... no llegaremos nunca a cambiar globalmente el mundo en que vivimos. La puesta en marcha de acciones como las anteriores, no resulta una fácil empresa, impedimentos como la inercia producida por años de un consumismo irracional y la necesidad de la renuncia a hábitos y formas de producción incongruentes con una mentalidad ambientalista, hacen que se haga necesario una gran voluntad política para su ejecución. Aunque no existen fórmulas mágicas para alcanzar estas aspiraciones, sí que se pueden plantear estrategias metodológicas operativas que a corto y medio plazo nos acerquen progresivamente a unas formas de vida más respetuosas con el entorno que nos rodea. Partiendo de la idea de que la educación está institucionalizada en las escuelas, en los institutos y en las universidades, bien podían estas instituciones tener una mayor responsabilidad en la divulgación de valores coherentes con el entorno. Las decisiones no sólo deberían afectar a la incorporación de programas de Educación Ambiental dentro de los currículum, las propias instituciones de educación superior deben estar diseñadas para transmitir este tipo de mensajes en su arquitectura, en el uso modélico que hacen de sus recursos, en la planificación, el manejo y mantenimiento de zonas verdes y la adecuación de los espacios de ocio que rodean al centro o institución educativa.

Las universidades deben jugar un papel mucho más activo en el proceso de transición hacia las sociedades sostenibles, en razón del peso que poseen en la formación profesional, la investigación científica y la difusión de la cultura en las sociedades contemporáneas. Estamos asistiendo a un rápido crecimiento del número de jóvenes que acceden a los sistemas de Educación Superior, este hecho convierte a las universidades en escenarios privilegiados para la construcción de modelos sociales y económicos cada vez más sostenibles. El lugar que han de ocupar estos nuevos profesionales en los escenarios laborales del futuro constituyen una fuerza de cambio importante para afrontar los retos del desarrollo sostenible a escala planetaria.

En este sentido apunta la filosofía de formación de redes universitarias hacia la sostenibilidad, cuyas principales metas se inspiran en principios como los siguientes: a) Rescatar el espíritu crítico de la formación universitaria para que asuma su función social en un ambiente de libertad. b) Incorporar y potenciar la dimensión ambiental en las distintas disciplinas universitarias y en los diferentes niveles de la educación superior. c) Integrar las diferentes disciplinas para afrontar los problemas de desarrollo y sostenibilidad desde la interdisciplinariedad. d) Propiciar nuevas estructuras, dentro de las universidades, que tengan como objetivo concreto la problemática ambiental. e) Impulsar la investigación de tecnologías apropiadas a las condiciones territoriales de contexto y coordinar esfuerzos de trabajo en red. f) Implementar los aspectos de ecoeficiencia y suficiencia en bs proyectos de investigación desarrollados conjuntamente con las empresas. g) Desarrollar estrategias de sostenibilidad integradas dentro de cada 
universidad y su entorno social, que actúen como dinamizadores del cambio de mentalidad necesario para afrontar la crisis actual de la civilización industrial.

Según Benayas (2003): "La máxima piensa globalmente, actúa localmente instaura el poder de transformación desde las actividades más corrientes, desde el lugar en el se vive, trabaja y estudia para ayudar a conservar un planeta amenazado". En sentido general, los distintos niveles de enseñanza, desde la educación primaria hasta la universitaria, han respondido de algún modo a este reclamo. Entonces, ambientalizar la universidad no es una consigna a tono con los momentos actuales, es una necesidad si tenemos en cuenta que en ella estudian y se forman los decisores del mañana. A lo largo de los últimos años, diferentes universidades han creado departamentos de gestión ambiental, comisiones y órganos de participación específica ligada a los problemas del medio ambiente. Tampoco es ajena la preocupación que públicamente vienen manifestando los principales responsables de la Educación Superior, ya sean ministros de educación, ciencia y tecnología, o rectores universitarios en las Conferencias periódicas a nivel mundial; en las que una y otra vez se viene reiterando la necesidad de instrumentalizar estrategias y acciones de distinto nivel y alcance en relación a las temáticas ambientales. Acciones en las que se trata de dar respuesta al Plan denominado "Agenda 21", aprobado por líderes de 179 países en la Cubre de la Tierra de Río de Janeiro, en el que se establecía un llamamiento a las diferentes instituciones, administraciones y gobiernos para que aplicaran estrategias de desarrollo sostenible en sus respectivos ámbitos de intervención, recomendando encarecidamente la realización de procesos participativos que permitan alcanzar el consenso social para la mejora ambiental del entorno próximo. A tales propósitos responden iniciativas como las de la Organización Internacional de Universidades por el Desarrollo Sostenible y el Medio Ambiente, al plantearse para Europa y América Latina objetivos como los siguientes:

1) Tener mayor presencia institucional a través de medios de difusión especializados en las temáticas ambientales y redes de docencia e investigación conjunta entre países de Europa y Latinoamérica.

2) Promover proyectos interinstitucionales en áreas de interés conjunto, tanto en docencia e investigación como en las dimensiones de gestión y extensión universitaria.

3) Impulsar, a nivel más concreto, la realización de programas de doctorado conjuntos entre universidades ibéricas y latinoamericanas sobre los diferentes aspectos y dimensiones del desarrollo sostenible.

4) Establecer una comunicación permanente con organismos regionales afines como la OEI, así como con organismos nacionales o regionales de educación, medio ambiente y desarrollo sostenible.

5) Instar a las autoridades convocantes de ayudas y recursos (especialmente $\mathrm{AECl}, \mathrm{ICl}$ y $\mathrm{OEI}$ ) para que incorporen entre las líneas prioritarias de cooperación aquellas acciones o temáticas referidas directamente al desarrollo sostenible.

6) Sugerir a los diferentes órganos de coordinación científica de cada país en particular (CONACYT, CICYT,... ) y a las diferentes Redes Europeas y Latinoamericanas de Evaluación y Acreditación (RIACES, Red Iberoamericana para la Acreditación de la Calidad de la Educación Superior; ECA, Consorcio Europeo de Agencias de Acreditación, ENQA, Red Europea para la Garantía de la Calidad de la Educación Superior) que incorporen en sus formularios de 
concesión de proyectos de investigación y protocolos de concesión de certificación, acreditación y evaluación unas más altas exigencias y compromisos con la implantación de sistemas de gestión ambiental y construcción de metodologías de evaluación basadas en indicadores de sostenibilidad comparables.

Las estrategias de ambientalización universitaria se establecen básicamente en tres ámbitos: 1) Ambientalización curricular, consiste en la incorporación de la dimensión ambiental en los recorridos formativos, bien a través de la promoción de nuevos estudios interdisciplinares (tales como las licenciaturas en Ciencias Ambientales, los postgrado en Sistemas de Gestión Ambiental, o los doctorados en Medio Ambiente), con asignaturas específicas de medio ambiente o mediante la integración de la dimensión ambiental en los diferentes campos disciplinares. 2) Gestión ambiental sostenible, las universidades como organizaciones generan una serie de impactos sobre el medio y deben planificar actuaciones correctoras que eviten o minimicen esos impactos, mediante la implantación de los correspondientes sistemas de gestión ambiental y la planificación a corto, medio y largo plazo de actuaciones que involucren a toda la comunidad en el desarrollo de compromisos ambientales; entre las actuaciones principales que comprende el campo de la gestión ambiental sostenible podemos destacar la gestión de los residuos, el aumento de la autonomía y eficiencia energética mediante el uso de energías renovables y el ahorro energético y de agua, el diseño y la ordenación de las infraestructuras y la incorporación de criterios ambientales en los edificios, contratas y proveedores. 3) Educación y participación ambiental, parte de la idea de que la ambientalización universitaria pasa por ofrecer oportunidades para que los estudiantes incorporen en sus estilos de vida y en sus códigos deontológicos profesionales principios y fundamentos inspirados en las buenas prácticas proambientales.

\section{PRECEDENTES INNOVADORES Y EXPERIENCIAS INSTITUCIONALES DE BUENAS PRÁCTICAS AMBIENTALES EN EL CONTEXTO UNIVERSITARIO}

La formación ambiental de los estudiantes adquiere una importancia capital, por cuanto, como hemos señalado anteriormente, son ellos los que muy pronto serán responsables de la toma de decisiones en las más disímiles esferas de la sociedad. Sin embargo, los enfoques predominantes no siempre abordan medidas de integración global de los modelos de aseguramiento de la calidad ambiental en la gestión rutinaria de sus instituciones. En el mejor de los casos, dichos modelos ponen énfasis en el control de riesgos y en la minimización de residuos y gastos energéticos, especialmente en los campos de formación universitaria de corte más científico, mientras se ha visto restringida la ejecución de algunas acciones principalmente en el ámbito puramente curricular en algunas disciplinas de corte más Humanístico y ligado a las Ciencias Sociales. Si bien una creciente preocupación por integrar temáticas medio ambientales en los programas formativos empieza a dar sus frutos gracias a la constitución de Redes Universitarias de ámbito internacional y al intercambio de buenas prácticas en el aseguramiento de la calidad ambiental en los entornos de Educación Superior.

La introducción de la problemática ambiental en el currículo y gestión universitaria, ha transitado durante décadas con diferentes grados de profundidad, amplitud y éxitos en su aplicación. Las experiencias revisadas denotan que el tratamiento dado a la problemática ambiental abarca un diapasón que va desde el tratamiento informativo de temas puramente ecológicos, hasta abarcar esta temática de un modo global, tocando tanto aspectos curriculares como de gestión universitaria, pero sobre todo, con una estrategia 
participativa. A continuación trataremos de resumir mediante un cuadro algunas experiencias del contexto de Latinoamérica y Europa. Señalando en primer lugar los proyectos, misiones y resultados de las principales Redes Ambientes de Educación Superior preocupadas por la ambientalización y el aseguramiento de la calidad ambiental de sus instituciones, pasando en segundo lugar a describir algunos casos específicos de universidades comprometidas con el medio ambiente.

Cuadro 1.

Redes Europeas y Latinoamericanas preocupadas por el aseguramiento de la calidad ambiental en las instituciones de Educación Superior

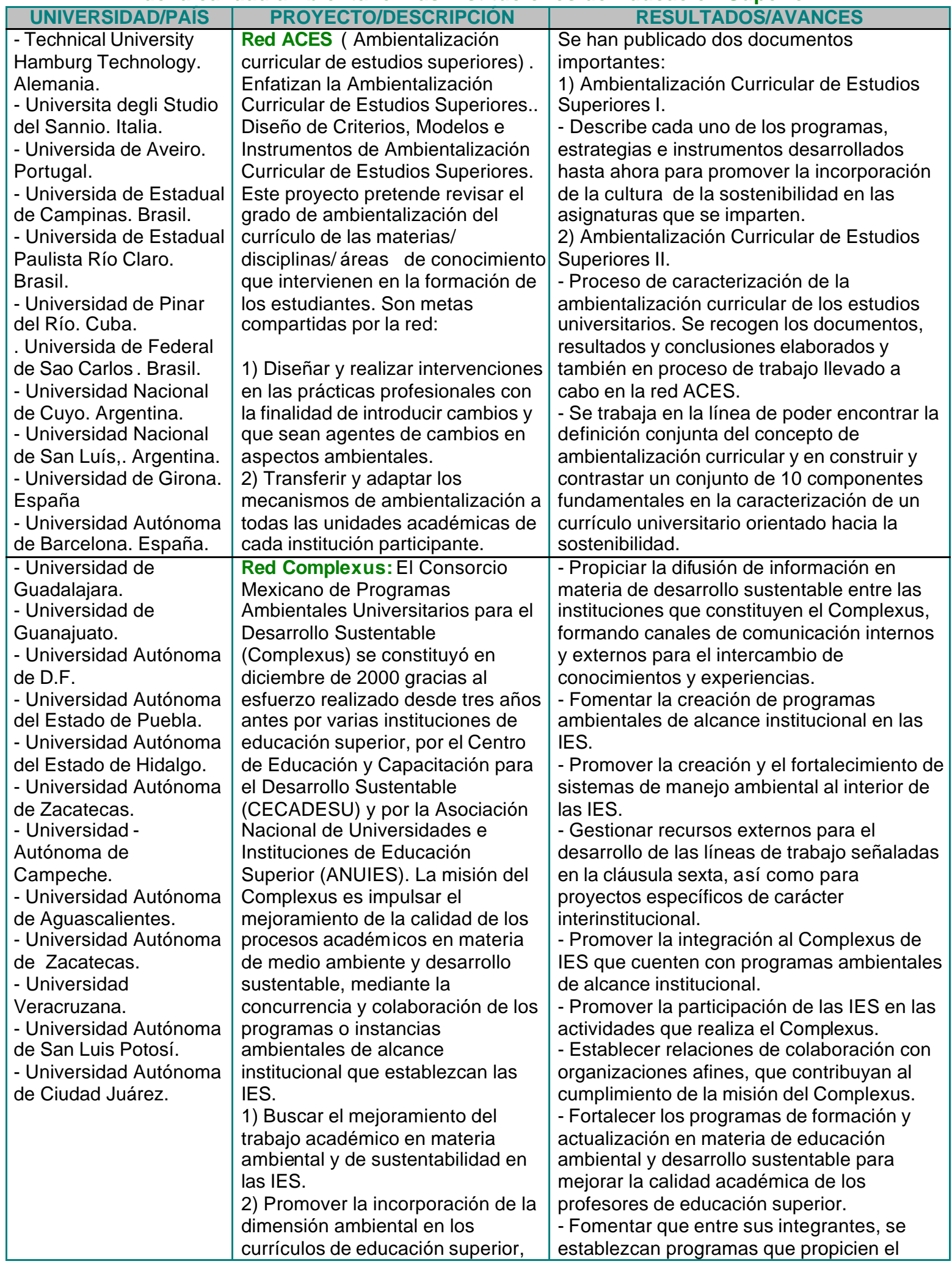




\begin{tabular}{|c|c|c|}
\hline & $\begin{array}{l}\text { así como la elaboración e } \\
\text { intercambio de propuestas teóricas } \\
\text { y metodológicas que tengan ese } \\
\text { propósito. }\end{array}$ & $\begin{array}{l}\text { desarrollo de conocimientos, aptitudes, } \\
\text { competencias, habilidades, valores y } \\
\text { actitudes necesarias en materia del } \\
\text { desarrollo sustentable. }\end{array}$ \\
\hline $\begin{array}{l}\text { Esta red acoge a más } \\
\text { de } 60 \text { universidades } \\
\text { latinoamericanas y } \\
\text { españolas }\end{array}$ & $\begin{array}{l}\text { Red OIUDSMA: Organización } \\
\text { Internacional de Universidades por } \\
\text { el Desarrollo Sostenible y el Medio } \\
\text { Ambiente. Constituida en } 1997 . \\
\text { Es una red cuya finalidad se centra } \\
\text { en el desarrollo de programas } \\
\text { docentes e investigadores en el } \\
\text { campo del medio ambiente y el } \\
\text { desarrollo sostenible. } \\
\text { Constituyen prioridades de trabajo } \\
\text { de la red: } \\
\text { Establecimiento de redes de } \\
\text { información internacionales que } \\
\text { vinculen los sistemas nacionales, } \\
\text { subregionales, regionales e } \\
\text { internacionales. } \\
\text { Apoyo y fomento del acceso a la } \\
\text { transferencia de tecnología. } \\
\text { Apoyo a los programas de } \\
\text { cooperación y asistencia. } \\
\text { Establecimiento de redes de } \\
\text { colaboración entre centros de } \\
\text { investigación ambiental. } \\
\text { Favorecer el trabajo interdisciplinar } \\
\text { y el fomento de las experiencias de } \\
\text { investigación y su interpretación y } \\
\text { divulgación, permitiendo una toma } \\
\text { de decisiones en los ámbitos de la } \\
\text { política, lo social y lo tecnológico. } \\
\text { Integración de los conocimientos } \\
\text { científicos derivados de la } \\
\text { investigación sobre el medio } \\
\text { ambiente en las políticas } \\
\text { económicas y sociales. } \\
\text { Favorecer las metodologías que } \\
\text { permitan contextualizar cada } \\
\text { problema ambiental en su escala } \\
\text { real, evitando tanto un excesivo } \\
\text { localismo, así como una visión } \\
\text { excesivamente globalizadora que } \\
\text { impida una solución real de los } \\
\text { problemas. } \\
\text { Asumir compromisos de } \\
\text { divulgación de problemas, } \\
\text { conflictos y denuncia ambiental. }\end{array}$ & 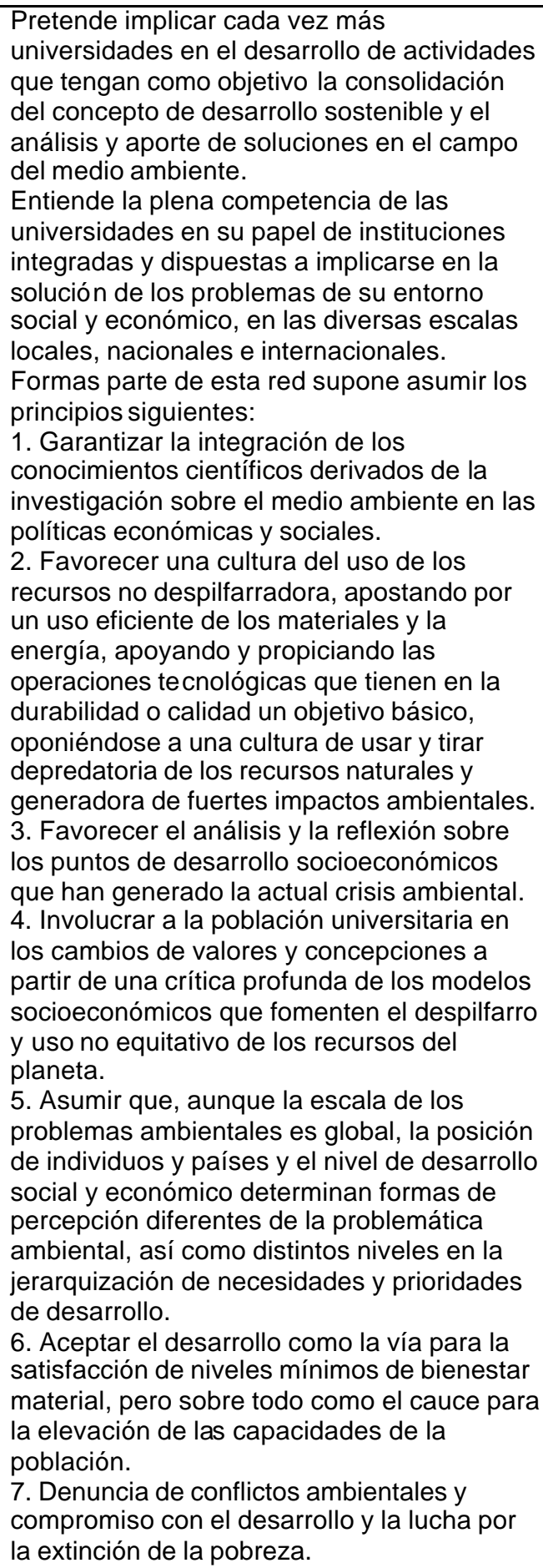 \\
\hline $\begin{array}{l}\text { Universidad de Santiago } \\
\text { de Chile }\end{array}$ & $\begin{array}{l}\text { Red SUMA } 21 \text { (Sustentabilidad y } \\
\text { Medio Ambiente para el siglo XXI). } \\
\text { Es una unidad de gestión que } \\
\text { desarrolla y conceptualiza } \\
\text { conocimiento ambiental y valida } \\
\text { metodologías propias para poner al } \\
\text { alcance de académicos, } \\
\text { profesores, estudiantes e } \\
\text { instituciones educativas recursos } \\
\text { para lograr protección ambiental } \\
\text { con sustentabilidad. }\end{array}$ & $\begin{array}{l}\text { Ha alcanzado un gran reconocimiento en } \\
\text { Chile por su liderazgo, amplia experiencia y } \\
\text { colaboración en el desarrollo de recursos } \\
\text { educacionales, programas de capacitación } \\
\text { profesional e investigación en ciencia } \\
\text { ambiental aplicada. Introduce el concepto de } \\
\text { ciudad ambiental bajo el cual se accede a los } \\
\text { grandes temas ambientales en plataforma } \\
\text { Internet con un portal que incluye recursos } \\
\text { didácticos relacionados con el medio } \\
\text { ambiente ( ENVIROEDUCA y prototipos para } \\
\text { la Química sustentable (GREEN } \\
\text { CHEMISTRY ), desarrollo sostenible, gestión } \\
\text { ambiental, análisis y monitoreo ambiental y }\end{array}$ \\
\hline
\end{tabular}




\begin{tabular}{|c|c|c|}
\hline & & control de la calidad del aire. \\
\hline $\begin{array}{l}\text { Centro de Información, } \\
\text { Gestión y Educación } \\
\text { Ambiental (CIGEA). } \\
\text { Cuba }\end{array}$ & $\begin{array}{l}\text { Red Cubana de Formación } \\
\text { Ambiental. Esta red está formada } \\
\text { por los órganos de la } \\
\text { administración central del estado, } \\
\text { las universidades, los centros de } \\
\text { capacitación e investigación y las } \\
\text { comunidades. Ha permitido dar a la } \\
\text { formación ambiental un carácter de } \\
\text { sistema. } \\
\text { Es un importante instrumento de } \\
\text { gestión ambiental para dar } \\
\text { respuesta a la demanda de } \\
\text { formación y capacitación con } \\
\text { nuevos enfoques holísticos e } \\
\text { interdisciplinarios y el } \\
\text { establecimiento de vínculos } \\
\text { interinstitucionales. }\end{array}$ & $\begin{array}{l}\text { La problemática ambiental es tratada en las } \\
\text { universidades cubanas a través de ejes } \\
\text { transversales y con una eficiencia que varía } \\
\text { no sólo entre la universidades del país, sino } \\
\text { también dentro de las distintas asignaturas y } \\
\text { disciplinas de una propia universidad. } \\
\text { Sin embargo no existe ningún plan de } \\
\text { ambientalización global como el que nos } \\
\text { proponemos que abarque tanto el plano } \\
\text { curricular como el extracurricular y el de } \\
\text { gestión universitaria. }\end{array}$ \\
\hline
\end{tabular}

La participación de las diferentes universidades en estas redes permite intercambiar experiencias y compartir proyectos conjuntos que aspiran a implantar una cultura de la calidad que da cabida y prioridad a las temáticas ambientales. Si bien la singularidad con que cada institución desarrolla sus acciones y proyectos en el entorno local, bien merece una mención específica, destacando algunos de los casos de más tradición:

\section{Cuadro 2.}

Ejemplos de buenas prácticas de aseguramiento de la calidad ambiental

\begin{tabular}{|c|c|c|}
\hline UNIVERSIDAD/PAIS & PROYECTO/DESCRIPCION & RESULTADOS/AVANCES \\
\hline $\begin{array}{l}\text { Universidad Estatal a } \\
\text { Distancia de San José } \\
\text { de Costa Rica. Costa } \\
\text { Rica }\end{array}$ & $\begin{array}{l}\text { Esta investigación analiza algunos } \\
\text { cursos de la carrera Ciencias de la } \\
\text { Educación I y II ciclos para } \\
\text { determinar en qué medida pueden } \\
\text { introducir elementos conducentes a } \\
\text { su ambientalización y dar } \\
\text { recomendaciones a la formulación } \\
\text { de una propuesta que favorezca el } \\
\text { desarrollo de los valores } \\
\text { ambientales. }\end{array}$ & $\begin{array}{l}\text { Se logró categorizar las descripciones de } \\
\text { objetivos que debe procurar un curso } \\
\text { ambientalizado y las conductas que se } \\
\text { esperan, tenga el individuo ambientalizado. } \\
\text { Los resultados obtenidos son válidos y } \\
\text { preliminares para nuevas investigaciones }\end{array}$ \\
\hline $\begin{array}{l}\text { Universidad de San Luis } \\
\text { de Potosí. México }\end{array}$ & $\begin{array}{l}\text { La Agenda Ambiental de la UASLP } \\
\text { es un mecanismo de coordinación } \\
\text { y colaboración horizontal entre } \\
\text { entidades académicas y } \\
\text { administratrvas. Su misión es } \\
\text { contribuir a la integración de la } \\
\text { perspectiva ambiental y del } \\
\text { desarrollo sostenible en todo el } \\
\text { quehacer de la Universidad, con la } \\
\text { participación de la comunidad } \\
\text { universitaria estudiantil, académica } \\
\text { y administrativa, de manera que } \\
\text { tenga un profundo impacto tanto en } \\
\text { el interior como en el exterior de la } \\
\text { institución. }\end{array}$ & $\begin{array}{l}\text { Entre las áreas de intervención específica } \\
\text { destacamos: } \\
\text { 1) Implantación de Sistemas de Gestión } \\
\text { Integral de Calidad Ambiental en las } \\
\text { instalaciones universitarias, ámbitos de } \\
\text { gestión y desarrollos curriculares. } \\
\text { 2) La prevención y control de riesgos } \\
\text { ambientales. } \\
\text { 3) Búsqueda de soluciones ope rativas a } \\
\text { problemáticas específicas del entorno } \\
\text { inmediato. } \\
\text { 4) El desarrollo de programas de posgrado } \\
\text { multidisciplinares en ciencias ambientales. }\end{array}$ \\
\hline $\begin{array}{l}\text { Universidad Autónoma } \\
\text { de Madrid. España }\end{array}$ & $\begin{array}{l}\text { Posee un Vicerrectorado específico } \\
\text { de Calidad Ambiental del Campus, } \\
\text { una oficina EcoCampus que } \\
\text { gestiona varios proyectos de } \\
\text { ambientalización y voluntariado y } \\
\text { dispone de una carta de } \\
\text { compromiso ambiental con la } \\
\text { Agenda } 21 . \\
\text { Entre las objetivos prioritarios de } \\
\text { su trabajo destacan: } \\
\text { 1. Ofrecer a la sociedad un ejemplo } \\
\text { de reflexión y preocupación }\end{array}$ & $\begin{array}{l}\text { Las acciones específicas desarrolladas para } \\
\text { dar respuesta a los objetivos son: } \\
\text { 1. Disminución de los niveles de emisión de } \\
\text { CO2 a la atmósfera derivados principalmente } \\
\text { de la gran movilidad universitaria, mediante } \\
\text { la promoción del transporte público y la } \\
\text { disminución del número de vehículos } \\
\text { privados que acceden al campus de la UAM. } \\
\text { 2. Sustitución progresiva y eliminación de } \\
\text { todos los productos utilizados en los } \\
\text { laboratorios y demás instalaciones del } \\
\text { campus que dañan la capa de ozono. }\end{array}$ \\
\hline
\end{tabular}




\begin{tabular}{|c|c|c|}
\hline & $\begin{array}{l}\text { ambiental acerca de las } \\
\text { consecuencias de las actividades } \\
\text { cotidianas. } \\
\text { 2. Promover fórmulas para } \\
\text { solucionar o mejorar los conflictos } \\
\text { ambientales que genera la propia } \\
\text { universidad en sus actividades } \\
\text { ordinarias. } \\
\text { 3. Incrementar de los procesos de } \\
\text { reducción, reciclaje y reutilización } \\
\text { de residuos. Incorporación de los } \\
\text { servicios de cafetería y } \\
\text { restaurantes a un plan estratégico } \\
\text { de recogida de residuos. } \\
\text { 4. Desarrollar metodologías de } \\
\text { evaluación adecuadas para la } \\
\text { implantación de auditorías } \\
\text { ambientales periódicas } \\
\text { encaminadas a la implantación y } \\
\text { normalización de buenas prácticas } \\
\text { en la actividad diaria del campus. }\end{array}$ & $\begin{array}{l}\text { 3. Consideración de criterios ambientales en } \\
\text { el diseño de los nuevos edificios e } \\
\text { instalaciones universitarias, mediante } \\
\text { soluciones energéticas alternativas, } \\
\text { arquitecturas bioclimáticas y selección de } \\
\text { materiales de construcción menos } \\
\text { contaminantes. } \\
\text { 4. Promoción de la biodiversidad en los } \\
\text { campus mediante la selección de especies } \\
\text { vegetales autóctonas. } \\
\text { 5. Gestión integrada de residuos tóxicos y } \\
\text { peligrosos, biológicos y radioactivos. } \\
6 \text {. Aplicación de criterios ambientales en la } \\
\text { evaluación de proveedores y empresas de } \\
\text { servicios. } \\
\text { 7. Disminución progresiva del consumo de } \\
\text { agua y energía. } \\
\text { 8. Incentivación de la venta y utilización de } \\
\text { modalidades de comercio justo. } \\
\text { Difundir entre los universitarios el } \\
\text { conocimiento ambiental de sus propias } \\
\text { actividades. }\end{array}$ \\
\hline $\begin{array}{l}\text { Universidad de } \\
\text { Granada. España }\end{array}$ & $\begin{array}{l}\text { Dispone de un Gabinete de } \\
\text { Calidad Ambiental desde donde se } \\
\text { coordina un Plan Estratégico de } \\
\text { Ambientalización Curricular y } \\
\text { Calidad Ambiental } \\
\text { Este plan incluye: } \\
\text { 1.Ecoauditorías a las actividades } \\
\text { de la universidad en sus diferentes } \\
\text { campus. } \\
\text { 2.Proyecto de rendimiento y ahorro } \\
\text { energético y de recursos. } \\
\text { 3.Programa de minimización de } \\
\text { residuos e impactos negativos. } \\
\text { 4.Incentivo y asesoramiento en la } \\
\text { ambientalización curricular. } \\
\text { 5.Sensibilización de la comunidad } \\
\text { universitaria. } \\
\text { 6.Integración de las interacciones } \\
\text { entre las dinámicas urbanas y las } \\
\text { de la universidad. } \\
\text { 7.Refuerzo de los efectos urbanos. } \\
\text { 8. Control de emisiones } \\
\text { atmosféricas. } \\
\text { 9. Evaluación de proveedores. } \\
\text { 10. Control de calidad acústica. } \\
\text { 11. Estudios de movilidad } \\
\text { estudiantil } \\
\text { 11. Información y sensibilización } \\
\text { ambiental. } \\
\text { 12. Formación ambiental. } \\
\text { 13. Apoyo a la investigación } \\
\text { ambiental }\end{array}$ & $\begin{array}{l}\text { Este proyecto es más amplio y abarcador, } \\
\text { pues a diferencia de otros anteriores, cumple } \\
\text { con uno de los requisitos fundamentales de } \\
\text { la educación ambiental, nos referimos a su } \\
\text { carácter participativo. } \\
\text { Por otra parte, abarca los aspectos } \\
\text { curriculares y extracurriculares, para ser } \\
\text { consecuente con el concepto de } \\
\text { Ambientalización en lo curricular y en la } \\
\text { gestión. } \\
\text { Los ingrediente fundamentales del Plan } \\
\text { Estratégico se inspiran en una definición } \\
\text { clara y precisa de la Política Ambiental de la } \\
\text { Universidad tratando de implantar un } \\
\text { Sistema Integral de Gestión Ambiental } \\
\text { definido conforme a los requisitos de las } \\
\text { Normas ISO 14 mil uno, asumiendo los } \\
\text { contenidos de la Conferencia de las } \\
\text { Naciones Unidas sobre Medio Ambiente y } \\
\text { Desarrollo (CNUMAD) y la responsabilidad } \\
\text { de llevar a cabo la transmisión y expansión } \\
\text { del conocimiento científico y tecnológico bajo } \\
\text { los principios de solidaridad intercultural e } \\
\text { intergeneracional. Las directrices de la } \\
\text { Política Ambiental quedan reflejadas en los } \\
\text { siguientes principios: } \\
\text { - Prevenir, reducir y eliminar cuando sea } \\
\text { posible la afección ambiental que pueda } \\
\text { derivarse de sus actividades como } \\
\text { organización } \\
\text { - Cumplir las disposiciones legales que le } \\
\text { afecten en materia ambiental y mantener una } \\
\text { relación de diálogo y colaboración con los } \\
\text { Organismos Ambientales competentes y las } \\
\text { instituciones del entorno social. }\end{array}$ \\
\hline $\begin{array}{l}\text { Universidad Politécnica } \\
\text { de Cataluña. } \quad \text { España }\end{array}$ & $\begin{array}{l}\text { Plan de Medio Ambiente de la } \\
\text { Universidad Politécnica de } \\
\text { Cataluña. Este plan se ejecuta en } \\
12 \text { escuelas, } 3 \text { facultades y } \\
\text { comprende } 41 \text { proyectos de acción } \\
\text { durante } 5 \text { años. Su objetivo es } \\
\text { ambientalizar el conjunto de } \\
\text { actividades de la universidad, es } \\
\text { decir, trabajar en defensa del } \\
\text { medio ambiente de una manera } \\
\text { transversal en todos los ámbitos: } \\
\text { formación, postgrado, } \\
\text { investigación, vida universitaria y }\end{array}$ & $\begin{array}{l}\text { Este plan está dirigido a toda la población } \\
\text { universitaria: estudiantes, profesores, } \\
\text { directivos y personal administrativo y de } \\
\text { servicios. } \\
\text { El mismo promoverá el desarrollo de un } \\
\text { modelo integrador del medio ambiente en la } \\
\text { universidad y proyectarlo en la sociedad } \\
\text { como contribución para la consecución de un } \\
\text { desarrollo sostenible. }\end{array}$ \\
\hline
\end{tabular}




\begin{tabular}{|l|l|l|}
\hline & sensibilización. & \\
\hline $\begin{array}{l}\text { Universidad Nacional de } \\
\text { San Luis. Argentina }\end{array}$ & $\begin{array}{l}\text { Diagnóstico Preliminar del Grado } \\
\text { de Ambientalización Curricular de } \\
\text { la Universidad Nacional de San } \\
\text { Luis. }\end{array}$ & $\begin{array}{l}\text { Este proyecto es también un proyecto amplio } \\
\text { y consecuente con el concepto de Educación } \\
\text { Ambiental pues sus acciones se orientan a } \\
\text { favorecer el desarrollo de un profesional que } \\
\text { adquiera una profunda comprensión sobre la } \\
\text { problemática ambiental en toda su } \\
\text { complejidad ( aspectos naturales, sociales } \\
\text { culturales, de salud, etc ) y asuma un fuerte } \\
\text { compromiso con el desarrollo sostenible, } \\
\text { entendido desde una perspectiva crítica que } \\
\text { contribuya a una sociedad cada vez más } \\
\text { justa y solidaria }\end{array}$ \\
\hline
\end{tabular}

Cada vez son más las universidades que han incorporado la variable ambiental en su gestión, en su docencia e investigación y, en general, en la vida universitaria. Las razones por las que de un tiempo a esta parte las universidades han emprendido actuaciones proambientales son: de carácter interno, el ahorro de costes, el aumento de la seguridad e higiene laboral, la demanda por parte de miembros universitarios o la promoción de buenas prácticas; externas, el cumplimiento de la legislación ambiental, los requerimientos de los gobiernos o de las instituciones financiadoras y, en menor medida, la presión de grupos ambientales. Comprometerse con el deterioro ambiental e iniciar actuaciones relacionadas con la gestión de residuos o la educación ambiental no dejan de ser una opción ética de cada institución universitaria, que pretende con ellas convertirse en un centro modélico de desarrollo ambientalmente sostenible para otras instituciones públicas y privadas (Benayas, 2003).

\section{PROPUESTA EMPÍRICA PARA EL ESTUDIO DE CASO Y VALIDACIÓN DEL MODELO DE ANÁLISIS DIAGNÓSTICO DE ASEGURAMIENTO DE LA CALIDAD AMBIENTAL}

El estudio de caso que nos ocupa trata de responder a un conjunto de necesidades sentidas, que se concretan en las siguientes: 1) La Universidad de Ciego de Ávila cuenta con una política ambiental bien definida y consecuente con la protección del medio ambiente, sin embargo, su gestión no responde a un enfoque ambientalista. 2) La ambientalización curricular es escasa y sólo se percibe claramente en algunas disciplinas impartidas en la carrera de Ciencias Agropecuarias y Mecanización de la Producción Agropecuaria. 3) La formación ambiental de los estudiantes de la Universidad de Ciego de Ávila es deficiente excepto los de la carrera de Ciencias Agropecuarias y Mecanización de la Producción Agropecuaria.

Esta investigación surge como una necesidad de establecer una política ambiental global en la Universidad de Ciego de Ávila que abarque tanto lo curricular como lo extracurricular y la gestión. Nos hemos propuesto los siguientes objetivos:

1) Analizar la política ambiental de la Universidad de Ciego de Ávila y en qué grado implica toda la población, es decir, dirección, claustro, estudiantes y personal de administración y servicios y proponer líneas de acción que potencien la ambientalización universitaria.

2) Evaluar la calidad de la educación ambiental que reciben los estudiantes de la Universidad de Ciego de Ávila y proponer medidas que optimicen la ambientalización del currículum .

3) Determinar el grado de preocupación ambiental de los estudiantes y su relación con la formación ambiental recibida. 
4) Implantar un programa de ecoauditorías internas que involucre todas las estructuras universitarias y cuyos resultados sirvan de base para un plan de acciones y la toma de conciencia ambientalista.

Muestra. Hemos trabajado con toda la población, la cual está formada por todos los estudiantes de los cursos regulares diurnos de todas las carreras que se imparten en la universidad de Ciego de Ávila, las cuales son: Ciencias Agropecuarias (desde primero hasta quinto año), Contabilidad (desde primero hasta quinto año), Informática (desde primero hasta tercer año), Estudios Socioculturales (primero y segundo año), Mecanización de la Producción Agropecuaria (desde primero hasta quinto año), Licenciatura en Turismo (primer año).

Instrumentos. Instrumentos empleados en la recogida de datos: cuestionarios, entrevistas, foro de discusión, observaciones y fotografías.

Fiabilidad. En nuestro caso calculamos los valores del coeficiente alfa de Cronbach obteniendo un valor de 0.7 para la escala de preocupación ambiental y 0.9 para la escala de formación ambiental.

Validez. En nuestro caso, aplicamos un análisis factorial utilizando el paquete estadístico SPSS 10.0 para Windows hallándose cinco factores: 1. Preocupación Ambiental (ítems 1; 3; 7; $10 ; 15$ y 16 y 17 ); 2. Control no personal (ítems 2; 4; 5; 8 y 9); 3. Actitud irresponsable ante la contaminación ambiental (ítems 12 y 13); 4. Preocupación por la fauna (ítems 6 y 11); 5. Actitud irresponsable ante la industria (ítem 14).

\section{ANÁLISIS DE DATOS Y DISCUSIÓN DE LOS RESULTADOS}

A) valoraciones sobre preocupación ambiental

Se aplicó la escala de preocupación ambiental de Weigel \& Weigel (1978), con las adaptaciones de Aragonés y Amerigo (1991) a 248 estudiantes de la Universidad de Ciego de Ávila. Los resultados obtenidos fueron codificados otorgándoles un valor desde 1 (muy en desacuerdo) hasta 5 (muy de acuerdo).

Los resultados corroboran una alta preocupación de los estudiantes, tal como se ha constado en otros estudios previos de Hernández Sampieri (2003). Todos los estudiantes tienen una misma drección respecto a su actitud hacia el medio ambiente, o sea, todos en este caso pueden ser calificados como proambientalistas. Estos resultados son similares a los obtenidos por otros investigadores, aunque la segmentación de los análisis en carreras no coinciden totalmente. Explicamos este resultado, por la gran información que brindan los medios de comunicación.

Se observan diferencias en la intensidad con que manifiestan esa actitud pro- ambientalista. Los valores más altos en las puntuaciones globales de la escala con 68.3 y una media por ítem de 4.3, pertenecen a la carrera de Agronomía; mientras la menor, 64 y una media por ítem de 4; pertenecen a Estudios Socioculturales e Informática. Entre estos dos extremos, se encuentran las carreras de contabilidad (65.7);Turismo(65.3); y Mecanización (67.3) con medias por ítem de $4.1 ; 4.1$ y 4.2 respectivamente.

\section{B) formación ambiental}

También se evaluó la formación ambiental recibida durante la carrera, se encuestaron los estudiantes de quinto año de las carreras de Agronomía, Contabilidad y Mecanización de la Producción Agropecuaria, los estudiantes de segundo año de Estudios Socioculturales y tercero de Informática por ser 
los años más avanzados de estas nuevas carreras. Se aplicó una escala de tipo Likert estructurado en cuatro bloques y donde se pidió que cada ítem fuera evaluado en una escala desde el 1 hasta el 5 correspondiendo el valor 1 a nulo, el valor 2 a muy escaso, el valor 3 a regular, el valor 4 a bien y el valor 5 a muy bien. Para cada ítem, se sumaron las valoraciones dadas como buenas y muy buenas y se dividieron entre el número de estudiantes encuestados, de modo que expresaremos los resultados como \% de bien y muy bien.

B.1. Ambientalización del currículo.- Sólo los estudiantes de la facultad de Mecanización de la Producción Agropecuaria valoraron en gran medida como bueno o muy bueno el trabajo realizado con el Medio Ambiente a través de actividades curriculares $70.4 \%$ y en segundo lugar los estudiantes encuestados de la facultad de Ciencias Agrícolas con el $48.6 \%$. Hay que destacar que en ninguna de estas dos facultades, se reportaron valoraciones de nulas o muy escasas. Las restantes facultades no rebasaron el $30 \%$ de satisfacción e incluso la carrera de Estudios Socioculturales sólo alcanzó el 17.6\% de valoraciones buenas o muy buenas. En estas tres carreras, Contabilidad, Estudios Socioculturales e Informática si se reportaron valoraciones de nulas o muy escasas. Todo lo anterior nos indica que deben tomarse urgentes medidas en la ambientalización de los currículos de estas tres carreras tanto en el número de asignaturas que deben ser ambientalizadas como en los métodos que se siguen para llevar la Educación Ambiental hasta los estudiantes.

B.2. Desarrollo de habilidades para enfrentar la problemática medioambiental.- Las facultades de Mecanización de la Producción Agropecuaria 70.4\% y de Ciencias Agrícolas 50\% han logrado implicar sus carreras en la problemática medioambiental aunque por sus características la facultad de Ciencias Agrícolas tiene mucho campo en el cual trabajar y con algunas ideas y propuestas específicas. Puede convertirse la carrera Agronomía en la carrera puntera en lo que Educación Ambiental se refiere, ya que su campo de aplicación es propicio a ello. Las restantes carreras tienen ante sí una ardua tarea que va desde la planificación hasta la preparación de los decentes pasando por la concientización de los directivos correspondientes.

B.3. Metodología y evaluación de aspectos medioambientales.-Sigue observándose que las carreras de Mecanización de la Producción Agropecuaria 72.2\% y una media de 3.7 y Agronomía 54.7\% con una media de 3.6 son las que más han trabajado metodológica y conceptualmente con la problemática medioambiental. La carrera de Agronomía tiene amplias posibilidades de un trabajo interdisciplinario que establezca buenas prácticas medioambientales que haría dar un salto cualitativo en este indicador. Las demás carreras está prácticamente en cero y necesitan iniciar un trabajo arduo y recibir asesoría metodológica para ello. Ninguna de las restantes carreras rebasó el valor medio de tres puntos.

B.4. Desarrollo de capacidades para enfrentar problemas ambientales.- Reflexionar, percibir pensar y comprender son procesos mentales que sólo son posibles cuando se tiene una sólida formación con respecto a un tema determinado que incluye un sistema de conocimientos y el dominio de una metodología para abordar el tema. La Educación Ambiental, no escapa a estos planteamientos, por lo que no debemos extrañarnos que nuevamente las carreras de Mecanización de la Producción Agropecuaria 94.4\% y Agronomía $81.3 \%$ presenten grados de satisfacción elevados y que éstos sean preocupantemente bajos en las tres restantes carreras. No puede ser de otra manera, pues las carreras de Contabilidad, Estudios Socioculturales e Informática, el trabajo que viene realizándose es muy escaso o en el mejor de los casos se realiza pero de una manera formal que no llega a los estudiantes por lo que el resultado es el 
mismo. Estas facultades deben iniciar el próximo curso con un plan de medidas encaminadas a darle un vuelco total a la situación actual.

B.5. Grado de ambientalización global de la carrera.- Por último se aplica un solo ítem el cual pide a los estudiantes que valoraran de forma integral la formación ambiental recibida durante su carrera. Solo los estudiantes de Agronomía 81.3\% y Mecanización de la Producción Agropecuaria 88.8\% se sienten satisfechos por la formación ambiental recibida y una media de 3.9 y 4 respectivamente en sus valoraciones.

\section{CONCLUSIONES}

La formación ambiental de los estudiantes de la Universidad de Ciego de Ávila no es uniforme, mientras esta formación es buena en las carreras de Ciencias Agrícolas y Mecanización de la Producción Agropecuaria, es muy deficiente en el resto de las carreras. Las causas de estas deficiencias debemos analizarla desde diferentes ángulos.

1. Aspectos Curriculares.- Las carreras de Contabilidad, Estudios Socioculturales e Informática, tienen un número muy pequeño de asignaturas ambientalizadas o que al menos aborden el tema medioambiental. La profundidad con que son tratados los temas medioambientales no es adecuada incluso en la carrera de Ciencias Agrícolas. Ninguna ciencia en particular puede desarrollarse sin tener en cuenta su historia y conocer lo que ha pasado en lo que a medio ambiente se refiere. Éste sería un buen punto de partida para asegurarnos que no se repitan acciones realizadas en décadas pasadas y que estamos todavía pagando. Deben buscarse alternativas que aborden la crisis medioambiental actual por cuanto es el centro y motor impulsor que ha hecho que la Educación Ambiental tenga la importancia estratégica que hoy día posee. Es imprescindible que todo ciudadano tome conciencia de que la problemática medioambiental tiene una dimensión social expresada en la responsabilidad que ha tenido el hombre en la crisis actual como por el efecto boumerang de esta crisis y que son las relaciones socio-políticas-económicas las que deben ser transformadas si deseamos transformar el medio ambiente. Es importante también que todo ciudadano conozca la legislación vigente en general y la relacionada con el medio ambiente en particular, vivimos en un planeta civilizado y las leyes y normas establecidas deben ser conocidas y cumplidas. El claustro debe alternativas que divulguen estas leyes y normas, sobre todo, las relacionadas con nuestro entorno. No se aplican los conocimientos de medio ambiente al entorno, de manera sistemática, hablamos de la capa de ozono, mientras a nuestro lado, un grifo derrama agua indiscriminadamente, esto es absurdo, y debemos partir de la máxima transforma lo local y estarás contribuyendo a transformar lo global. Para cumplimentar lo anterior, debemos partir de trabajar en base a nuestro entorno, se desconocen los problemas medioambientales de nuestra universidad y de nuestra provincia y se habla entonces de los tiburones blancos de Australia. Consideramos necesaria la ambientalización de las asignaturas de todas las carreras pero fundamentalmente de las que se imparten en Contabilidad, Estudios Socioculturales e Informática y para ello debe trabajarse a nivel de colectivos de año y de asignaturas a la vez que se analice en las correspondientes comisiones de carreras de modo que se tracen pautas al respecto pues su importancia lo requiere. Se considera necesario el asesoramiento metodológico a estas facultades por parte de profesores experimentados y con experiencia en este campo.

2. Desarrollo de Habilidades y Competencias Ambientales- Conocer los problemas, entender como se implica cada cual con el medio ambiente desde el punto de vista profesional así como saber actuar 
ante problemas medioambientales, son habilidades que no se desarrollan en la mayoría de las carreras de la universidad de Ciego de Ávila y es esto prácticamente lo que define el problema de nuestra investigación por lo cual las habilidades anteriores deben constituir un objetivo de todas las asignaturas que se imparten en esta universidad.

\section{Metodología usada en el tratamiento del medio ambiente y su incorporación al sistema de}

evaluación.- Es deficiente el desarrollo metodológico relacionado con la educación ambiental, así como la inclusión de estos temas en el sistema de evaluación. Lo anterior confirma la necesidad de actividades metodológicas al efecto y el asesoramiento correspondiente. Excepto los estudiantes de Mecanización de la Producción Agropecuaria y de Ciencias Agrícolas, el resto no tienen capacidad de reflexionar con criterio, percibir su complejidad, pensar en posibles soluciones ni comprender el carácter complejo, todo, referido al medio ambiente. De seguir así, estaremos formando al profesional que como dije al principio, dentro de muy poco tiempo y algunos ahora mismo son decisores de nuestra sociedad y tendrán que asumir esas funciones con serias limitaciones para solucionar una de las crisis más grandes que el hombre ha tenido que enfrentar, la crisis medioambiental. Toda investigación que siga la metodología Investigación Acción debe concluir con un grupo de acciones encaminadas a la mejora de aquellos aspectos cuya deficiencia fue detectada. En nuestro caso, tal y como hemos señalado anteriormente, estas acciones se proponen como producto de la discusión y el consenso entre el grupo representante de la población universitaria y que incluye todos los estratos de la población, o sea, estudiantes, dirección, administrativos, claustro y personal de servicio. Estas acciones son las siguientes ${ }^{1}$ : a) Constituir el voluntariado medioambiental en el que participen todos los sectores de la población universitaria. b) Crear la Oficina Agenda 21 que coordine todas las acciones de carácter medioambiental dentro de la universidad. c) Establecer un sistema de clasificación de residuos sólidos en el campus. d) Reciclar todos los residuos que sean posibles dentro o fuera de la universidad. e) Establecer un sistema de ecoauditorías internas con el objetivo de dar seguimiento a la situación medioambiental y proponer nuevas acciones para erradicar las deficiencias y que valore los siguientes indicadores: Uso y ahorro de agua, Uso y ahorro de energía eléctrica, iluminación, ventilación de aulas, laboratorios, biblioteca y dormitorios, Clasificación de residuos sólidos, Evacuación y tratamiento de residuales líquidos, Contaminación por escapes y ruido de vehículos automotores, Estado de espacios abiertos, Uso del tiempo libre, Barreras arquitectónicas, Forestación, Calidad de la nutrición, Hábitos de consumo, Establecer un sistema de buenas prácticas medioambientales en el campus y promoverlas hacia la comunidad, Establecer un sistema de actividades encaminadas a la capacitación de todos los trabajadores de forma tal que puedan conservar, mantener y mejorar el medio ambiente desde sus puestos de trabajo, Estudiar la posibilidad del uso de energía eólica, fundamentalmente en la extracción de agua en algunas áreas de la universidad, Dar asesoría a los profesores de las facultades de Contabilidad, Informática, Licenciatura en Turismo y Estudios Socioculturales en Educación Ambiental, Trabajar en los espacios abiertos de las áreas del campus que se encuentran despobladas con la asesoría de especialistas.

\footnotetext{
${ }^{1}$ Este trabajo no sólo tiene continuidad, sino que es una necesidad de la universidad si quiere dar respuesta a la encomienda social que le ha sido dada y que consiste en formar un hombre integralmente formado. Hoy día sería absurdo hablar de integridad de la personalidad sin referirse a un componente relacionado con la educación ambiental. Sin embargo, esta labor puede encontrarse en su desarrollo con dos limitaciones importantes: en primer lugar la de la inercia de algunos factores que tendrán forzosamente que cambiar algunos estilos de vida; en segundo lugar, el hecho de que muchas de las acciones a emprender necesitan de finanzas que no siempre están disponibles, por lo que se hace necesario ser más audaces en la búsqueda de estos recursos, a la vez que todas las personas que están implicadas en la planificación del presupuesto de la universidad, deben ver la conservación del medio ambiente como un factor priorizado.
} 


\section{BIBLIOGRAFÍA}

ARAgonÉS, I. y AMÉRIGO, A. (1991): "Un estudio empírico sobre las actitudes ambientales". Revista de Psicología Social, Vol. 6(2), pp. 240-248.

BENAYAs Del ÁlAMO, J. (2004): La ambientalización de los campus universitarios. El caso de la Universidad Autónoma de Madrid. Iller. Congreso Andaluz de Educación Ambiental. Sevilla: Consejería de Educación de la Junta de Andalucía.

CAlvo, S., BenAyAs, J. Y GutiÉRREZ, J. (2002): "Learning in sustainable environments: the greening of higher education", en TILBURY, D. et al. (Eds.): Education and sustainability. Responding to the Global challenge. Gland, Switzerland: IUCN-The World Conservation Union.

Coya García, M. (2001): Ambientalización de la Universidad de Santiago de Compostela. Tesis Doctoral. Universidad de Santiago de Compostela. España.

GUILLÉN,F.C. (1996): "Educación, Medio Ambiente y Desarrollo Sostenible". Revista Iberoamericana de Educación No 11, 1996. Disponible en http://www.campus-oei.org/oeivirt/rie11a03.htm. Última revisión 21 de junio de 2004.

GUTIÉRREZ PÉREZ, J. (1995): La Educación Ambiental. Fundamentos teóricos, propuestas de transveralidad y orientaciones curriculares. Madrid: La Muralla.

GutiérReZ PÉREZ, J. (2004): "La Ambientalización de centros educativos como factor de calidad de la gestión de organizaciones". Simposium 5: La Ambientalización de los centros educativos. Ecoescuelas y ecouniversidades. Presentación Iller Congreso Andaluz de Educación Ambiental, Consejería de Educación de la Junta de Andalucía.

HERNÁNDEZ SAMPIERI, R. (2003): Metodología de la Investigación. México:Mc Graw Hill, 2003.

RodríGuez, J. et al. (1996): Propuesta de un Modelo Teórico para la Ambientalización de cursos de una carrera universitaria. Una experiencia piloto. Costa Rica: EUNED.

Rodriguez Gómez,G.; GIL FlORES,J. Y GARCíA JiméneZ,E. (1996). Metodología de la Investigación Cualitativa. Málaga. Aljibe.

WEIGEL, R. \& WEIGEL, J. (1978): "Environmental concern. The development of a measure". Environment \& Behavior 10, pp. 3-15. 


\title{
Contactar
}

Revista lberoamericana de Educación

\author{
Principal OEI
}

